\title{
Terminologia Embryologica y Placenta: Propuesta de Términos Embriológicos en Español
}

\author{
Terminologia Embryologica and Placenta: Proposal of Embryological Terms in Spanish
}

\author{
Ruth Prieto Gómez ${ }^{1}$ \& Nicolás Ernesto Ottone ${ }^{2,3}$
}

PRIETO, G. R. \& OTTONE, N. E. Terminologia Embryologica y placenta: Propuesta de Términos Embriológicos en español. Int. J. Morphol., 36(1):63-68, 2018.

RESUMEN: En el área de la embriología, y en relación al uso de Terminologia Embryologica (TE), existen términos que son utilizados y que no se corresponden con ésta última. Pero a esta situación clásica, desde el origen de Nomina Anatomica de Basilea en 1895, se suma la ausencia de términos embriológicos en TE y que son diariamente reconocidos y nombrados en la práctica clínica. Además, no existe aún traducción oficial al español de TE. El objetivo de este trabajo consistió en realizar una propuesta de términos en español correspondientes a los términos incluídos en Paraplacenta [E6.0.2.4.0.1.], Placenta [E5.11.3.1.1.0.5] y Anomaliae placentae [E6.0.2.5.1.0.1], a partir de Terminologia Embryologica (TE) publicada por el Federal International Programme on Anatomical Terminologies en 2013, y en la cual sólo se encuentra la traducción al idioma inglés. La importancia de todos los trabajos relacionados con el buen uso de las terminologías y su correcta traducción al idioma vernáculo, radica en que la aplicación de un lenguaje único y común permitirá una mejor y mayor difusión de las investigaciones en el área de las ciencias morfológicas.

PALABRAS CLAVE: Terminologia Embryologica; Placenta.

\section{INTRODUCCIÓN}

El desarrollo de las ciencias morfológicas, y en especial la embriología, en estos últimos tiempos ha sido considerable, y ha provocado una gran cantidad de publicaciones científicas donde se dan a conocer los resultados de diversas investigaciones.

En relación a la terminología, conocemos el trabajo realizado en Terminologia Anatomica e Histologica por el SILAT (Cruz et al., 2010; Losardo et al., 2010, 2015), pero hay escasa publicación de artículos sobre Terminologia Embryologica. Y en relación a la Terminologia Embryologica propuesta por la FIPAT (FIPAT, 2013), ésta se encuentra en latín, y sólo traducida al inglés, no existiendo una traducción al idioma español. Además, al revisar diferentes textos y artículos, en español, en el área de la embriología, se detecta la utilización de diferentes términos para identificar una misma estructura o proceso.
Cuando en 1895 en Basilea, se creó Nomina Anatomica, se estableció que el latin debía ser el idioma oficial a partir del cual realizar las traducciones a las distintas lenguas vernáculas, como así también la eliminación de epónimos, sinónimos y la asignación de un único término por estructura o proceso (Vasquez \& del Sol, 2015; Vargas et al., 2016; Ottone et al., 2017).

El objetivo de este trabajo es realizar una propuesta de términos en español para las estructuras del capítulo Adnexa embryonica et fetalia; Membranae extraembryonicae et fetales correspondientes a los términos incluídos en Paraplacenta [E6.0.2.4.0.1.], Placenta [E5.11.3.1.1.0.5] y Anomaliae placentae [E6.0.2.5.1.0.1], a partir de Terminologia Embryologica (TE) publicada por la FIPAT en 2013, y en la cual solo se encuentra la traducción al idioma inglés.

\footnotetext{
${ }^{1}$ Departamento de Pediatría y Cirugía Infantil, Facultad de Medicina, Universidad de La Frontera, Temuco, Chile.

${ }^{2}$ Laboratorio de Plastinación y Técnicas Anatómicas, CICO - Centro de Investigación en Ciencias Odontológicas, Facultad de Odontología, Universidad de La Frontera, Temuco, Chile.

${ }^{3}$ Doctorado en Ciencias Morfológicas, Facultad de Medicina, Universidad de La Frontera, Temuco, Chile.

Financiado por la Universidad de La Frontera, Proyecto DIUFRO Nº DI16-0074
} 


\section{MATERIAL Y MÉTODO}

Se analizaron los términos y procesos embriológicos de los capítulos de TE, correspondientes a los términos incluídos en Paraplacenta [E6.0.2.4.0.1.], Placenta [E5.11.3.1.1.0.5] y Anomaliae placentae [E6.0.2.5.1.0.1] (FIPAT). Además, se revisaron libros de texto y artículos científicos en idioma español con el propósito de verificar los términos embriológicos.

A partir de este análisis, se generaron cuatro tablas: Tabla I, correspondiente a la traducción al español de términos en latín existentes en TE; Tabla II, se incluyeron modificaciones de términos en latín existentes en TE y su correspondiente traducción al español; Tabla III, contiene, según nuestro análisis, términos agregados a TE, no existentes originalmente en la misma, con su correspondiente traducción al español; Tabla IV, en la que se incluyeron términos que deben ser eliminados de TE.

\section{RESULTADOS Y DISCUSIÓN}

Análisis de los Términos. En la tabla I están presentes los términos más frecuentemente utilizados en textos y artículos para la descripción de estructuras y procesos (Bernirschke \& Kaufmann, 2000; Uzcátegui et al., 2005; Apaza Valencia, 2006; Huppertz, 2008; Carlson, 2009; Bustamante-Zuluaga et al., 2011; Pérez Sánchez \& Donoso, 2011; Prieto Gómez et al., 2011; Roa et al., 2012; Sadler, 2012; Arteaga \& García, 2013; Dudek, 2014; Flores, 2015; Moore et al., 2016).

En la Tabla I se incluyeron los términos latinos cuya correlación morfológica es correcta, y por lo tanto, nos permitió realizar a partir de ellos la correspondiente traducción al español. Sin embargo, entre estos términos, existe la excepción del término Placenta discoidea [E6.0.2.5.0.1.3], el cual es de origen griego.

La mayoría de los términos son individuales, es decir, existe un término por estructura embriológica, como lo indica los principios de la TE. Sin embargo, hay tres excepciones, las cuales son: Placenta accessoria; Placenta succenturiata [E6.0.2.5.0.2.2]; Placenta bilobata; Placenta bipartite [E6.0.2.5.0.2.4]; Hydramnion; Polyhydramnion [E6.0.2.5.1.1.3]. En estos tres casos, las traducciones al español de los dos términos existentes en latín son utilizadas indistintamente, una u otra, para describir una misma estructura. En TE se recomienda la utilización de un solo término por estructu- ra. En estos casos, sugerimos una futura investigación con mayor profundidad para determinar cual de estos términos debe mantenerse y cual eliminarse.

En muchos de los términos en latín se mantienen las mismas palabras en español, utilizando el mismo término en latin para su correspondiente traduccióna al español, ya que poseen el mismo significado morfológico, como por ejemplo el término placente [E5.11.3.1.1.0.5], es utilizado en latín y español, sin realizar una traducción literal, que en este caso sería torta.

Otra situación que se observa en la Tabla I, es la correspondiente a los términos Placenta multilobata, Placenta multipartite [E6.0.2.5.0.2.7] y Placenta vallata, Placenta circumvallata [E6.0.2.5.0.2.9], en los cuales, existiendo dos términos en latín para una misma estructura, se seleccionó uno de ellos, el cual fue traducido al español, quedando, respectivamente, como Placenta multilobada y Placenta circumvallata. Esta selección se basó en que los términos que se mantuvieron son frecuentemente utilizados en la literatura y textos de la disciplina.

En la Tabla II, en negrita, se encuentran los nuevos términos en latin sugeridos en este trabajo, con su correspondiente traducción al español.

En el caso del término Membrane haemochorialis [E6.0.2.5.0.1.5], en este término, fue reemplazado la palabra Membrane por Placenta, y de esta manera, se transformó en Placenta haemochorialis, cuya traducción al español es Placenta hemocorial.

En el caso de los términos Gradus villosus initialis [E6.0.2.5.0.1.7], Gradus labyrintheus [E6.0.2.5.0.1.8], Gradus villosus definitivus [E6.0.2.5.0.1.9], se modificó a la actual clasificación de vellosidades primarias, secundarias y terciarias, respecticamente (Apaza Valencia; Huppertz), por lo cual los términos en latín fueron necesariamente modificados: Villitatis chorion prima, Villitatis chorion secunda, Villitatis chorion tertia, respectivamente.

Con respecto a los tres siguientes términos, relacionados con la zona de inserción del cordón umbilical, en latín solo aparece la ubicación de inserción pero sin mencionar que corresponde al cordón umbilical, además de que estos términos no se encontraban dependiendo de el término cordón umbilical, el cual a su vez se encuentra ausente en la TE. Por lo tanto, se procedió a incorporar en estos tres términos "funiculi umbilicalis", cuya traducción es cordón umbilical. 
Tabla I. Traducción al español de términos en latín existentes en Terminologia Embryologica.

\begin{tabular}{|c|c|c|}
\hline Código TE & Latín & Español \\
\hline E6.0.1.2.0.0.37 & Chorion Laeve & Corion liso \\
\hline E6.0.2.5.0.1.2 & Decidua parietalis & Decidua parietal \\
\hline E5.11.3.1.1.0.5 & PLACENTA & PLACENTA \\
\hline E6.0.2.5.0.1.1 & Insignia placentae humanae & Características de la placenta humana \\
\hline E6.0.2.5.0.1.3 & Placenta discoidea (griego) & Placenta discoidal \\
\hline E6.0.2.5.0.1.6 & Gradus formationis placentae & Estadios de formación de la placenta \\
\hline E6.0.2.5.0.2.1 & Formae placentae & Formas placentarias, Formas de la placenta \\
\hline E6.0.2.5.0.2.2 & $\begin{array}{l}\text { Placenta accessoria; } \\
\text { succenturiata }\end{array}$ & Placenta accesoria; Placenta succenturiata \\
\hline E6.0.2.5.0.2.3 & Placenta a nular is & Placenta anular \\
\hline E6.0.2.5.0.2.4 & Placenta bilobata; Placenta bipartite & Placenta bilobulada; plac enta bipartita \\
\hline E6.0.2.5.0.2.5 & Placenta lobata & Placenta lobulada \\
\hline E6.0.2.5.0.2.6 & Placenta membranacea & Placenta membranacea \\
\hline E6.0.2.5.0.2.7 & Placenta multilobata, Placenta multipartite & Placenta multilobulada \\
\hline E6.0.2.5.0.2.8 & Placenta trilobata & Placenta trilobulada \\
\hline E6.0.2.5.0.2.9 & Placenta vallata, Placenta circumvallata & Placenta circumvallata \\
\hline E6.0.2.5.0.3.1 & Varietates insertionis fu niculus umbilicalis & Variedades de inserción del cordón umbilical \\
\hline E6.0.2.5.0.4.9 & Placenta praevia marginalis & Placenta previa marginal \\
\hline E6.0.2.5.1.0.1 & Anomaliae membranarum fetalium & Anomalías de las membranas fetales \\
\hline E6.0.2.5.1.1.1 & Anomaliae amnii et liquoris amnio tici & Anomalias del amnios y líquido amniótico \\
\hline E6.0.2.5.1.1.3 & Hydramnion; Polyhydramnion & Hidramnios; Polihidramnios \\
\hline E6.0.2.5.1.1.4 & Oligohydramnion & Olihidramnios; Oligoamn ios \\
\hline E6.0.2.5.1.1.5 & Taenia amniotica & Brida amniótica \\
\hline E6.0.2.5.1.2.1 & Anomaliae chorii & Anomalías corió nicas \\
\hline E6.0.2.5.1.2.2 & Deformitas placentae & Deformidad placentaria \\
\hline E6.0.2.5.1.2.3 & Defectus placentae & Defecto placentario \\
\hline E6.0.2.5.1.2.4 & Defectus paraplacentalis chorii & Defecto coriónico placentario \\
\hline E6.0.2.5.1.3.2 & A. umbilicalis singularis & Arteria u mbilicar única \\
\hline E6.0.2.5.1.3.1 & Anomaliae funicul i umbilical is & Anomalías del cordón umbilical \\
\hline E6.0.2.5.1.3.6 & Nodus spurious funiculi ambilicalis & Nudo falso del cordón umbilical \\
\hline E6.0.2.5.1.3.7 & Nodus verus funiculi umbilicalis & Nudo verdadero del cordón umbilical \\
\hline E6.0.2.5.1.4.1 & Anomaliae placentae & Anomalias placentarias \\
\hline E6.0.2.5.1.4.2 & Placenta accreta & Placenta acreta \\
\hline E6.0.2.5.1.4.4 & Placenta extrachorialis & Placenta extracorial \\
\hline E6.0.2.5.1.4.5 & Placentafenestrata & Placenta fenestrada \\
\hline E6.0.2.5.1.4.6 & Placenta incarcerata & Placenta incarcerada \\
\hline E6.0.2.5.1.4.7 & Placenta increta & Placenta increta \\
\hline E6.0.2.5.1.4.8 & Placenta panduriformis & Placenta panduriforme \\
\hline E6.0.2.5.1.4.9 & Placenta percreta & Placenta percreta \\
\hline
\end{tabular}

E6.0.2.5.0.3.2 Insertio centralis $=$ Insertio centralis funiculi umbilicalis

E6.0.2.5.0.3.3 Insertio marginalis = Insertio marginalis funiculi umbilicalis

E6.0.2.5.0.3.4 Insertio velamentosa $=$ Insertio velamentosa funiculi umbilicalis

Por otro lado, existe el término Funiculus umbilicalis glomeratus [E6.0.2.5.1.3.3], pero este no identifica a la estructura cordón umbilical (inexistente en la TE en latín), por lo cual se procedió a eliminar la palabra glomeratus, que- dando solo como Funiculus umbilicalis, cuya traducción al español es cordón umbilical.

Con respecto al término Vesicula Allantoica [E6.0.2.5.1.3.8], éste como tal no identifica correctamente a la estructura correspondiente, por lo cual se procedió a modificar completamente el término, manteniendo la numeración y cambiándolo por Allantoides. La traducción al español de este término es Alantaoides.

En relación a los siguientes términos: 
Tabla II. Modificaciones de términos en latín existentes en Terminologia Embryologica. y su correspondiente traducción al español.

\begin{tabular}{|c|c|c|}
\hline Código $T E$ & Latín & Español \\
\hline E6.0.2.5.0.1.5 & Membrane haemochorialis = Placenta haemochorialis & Placenta hemocorial \\
\hline E6.0.2.5.0.3.2 & Insertio centralis = In sertio centralis funiculi umbilicalis & Inserción central del cordón um bilical \\
\hline E6.0.2.5.0.3.3 & Insertio marginalis = Insertio marginalis funic uli umbilicalis & Inserción marginal del cordón umbilical \\
\hline E6.0.2.5.0.3.4 & Insertio velamentosa $=$ In sertio velamentosa funiculi umbilicalis & Inserción velamentosa del cordón umbilical \\
\hline E1.0.2.6.3.0.6 & Placenta praevia $=$ Placenta praevia occlusiva & Placenta previa oclusiva \\
\hline E6.0.2.5.0.4.1 & Varietates situs placentae $=$ Varietates situs implantationis placentae & Variedades de sitio de implantación de la placenta \\
\hline E6.0.2.5.0.4.5 & Situs fundalis placentae $=$ Implantatio fundalis placentae & Implantación de la placenta en el fondo uterino \\
\hline E6.0.2.5.0.4.6 & Situs cornual is placentae $=$ Implantatio cornualis placentae & Implantación de la placenta en el cuerno \\
\hline E6.0.2.5.0.4.7 & Placenta praevia centralis $=$ Placenta praevia occlusive totalis & Placenta previa oclusiva total \\
\hline E6.0.2.5.0.4.8 & Placenta praevia lateralis = Placenta praevia occlusive partialis & Placenta previa oclusiva parcial \\
\hline E6.0.2.5.0.4.10 & Situs cervicalis placentae $=$ Implantatio cervicalis placentae & Implantación cervical de la placenta \\
\hline
\end{tabular}

[E6.0.2.5.0.4.5] Situs fundalis placentae = Implantatio fundalis placentae y [E6.0.2.5.0.4.6] Situs cornualis placentae = Implantatio cornualis placentae, se realizaron modificaciones en relación a la palabra situs, la cual es eliminada y cambiada por la palabra implantatio. La palabra "situs" se refiere a que "algo está sobre" y no expresa la idea de que algo "esté implantado", "incorporado" a otra estructura, como es en este caso.

En la Tabla III, se crearon nuevos términos en latín a partir de estructuras que poseían su correspondiente denominación en idioma español. Estos términos existentes en la literatura consultada en español no existían en la TE, por ello fueron agregados y traducidos al latin, como es el caso del término barrera placentaria, cuya traducción al latin propuesta en este trabajo es obex placentalis.

Se crearon dos términos en latín, en relación a situaciones clínicas (Uzcátegui et al.; Bustamante-Zuluaga et al.) que pueden presentarse, y cuya identificación es necesario determinar a partir de términos. En español, estos términos se definen como Circular de cordón umbilical reductible (Circumfuniculus umbilicalis reductibilis) y Circular de cordón umbilical irreductible (Circumfuniculus umbilicalis irreductibilis).

Tabla III. Términos creados y que deberían ser agregados a Terminologia Embryologica, ya que no existen originalmente en la misma, con su correspondiente traducción al español.

\begin{tabular}{ll}
\hline Latín & Español \\
\hline Obex placentalis & Barrera placentaria \\
Circumfuniculus umbilicalis reductibilis & Circular de cordón umbilical reductible \\
Circumfuniculus umbilicalis irreductibilis & Circular de cordón umbilical irreductible \\
\hline
\end{tabular}

Tabla IV. Términos de Terminologia Embryologica no encontrados en la revisión de textos y artículos.

\begin{tabular}{ll}
\hline Código TE & Latín \\
\hline E6 0.2.4.0.0.1 & PARAPLACENTA \\
E6.0.2.5.0.1.2 & Placenta deciduata \\
E6.0.2.5.0.1.4 & Vascularisatio chorioallan toica \\
E6.0.2.5.0.5.1 & Varietates cascularis ationis placentae \\
E6.0.2.5.0.5.2 & Vascularisatio dispersaplacentae \\
E6.0.2.5.0.5.3 & Vascularisatio magis tralis placentae \\
E6.0.2.5.1.1.2 & Adhaesio amnii \\
E6.0.2.5.1.3.4 & Strangulatio \\
E6.0.2.5.1.3.5 & Amputatio \\
E6.0.2.5.1.4.3 & Placenta adhaerens \\
E6.0.2.5.0.4.2 & Implantatio dorsalis placentae \\
E6.0.2.5.0.4.3 & Situs lateralis placentae \\
E6.0.2.5.0.4.4 & Situs ventralis placentae \\
\hline
\end{tabular}

Tabla V. Términos pertenecientes a otros capítulos que deben ser incluídos en los capítulos analizados en este trabajo.

\begin{tabular}{lcc}
\hline Código TE & Latín & Español \\
\hline E6.0.1.4.0.0.7 & Decidua & Decidua \\
E6.0.1.4.0.0.8 & Decidua basalis & Decidua basal \\
E6.0.1.4.0.0.15 & Decidua capsularis & Decidua capsula1 \\
E6.0.1.4.0.0.16 & Decidua parietalis & Decidua parietal \\
\hline
\end{tabular}

En los textos y artículos revisados sobre la temática, no fueron encontrados los términos presentes en la TE e incluídos en la Tabla IV. 
Tabla VI. Términos que deben ser eliminados y reemplazados por términos ya existentes en otros capítulos de Terminologia Embryologica

\begin{tabular}{|c|c|c|c|}
\hline \multicolumn{2}{|c|}{ Términos a eliminar } & Términos de reemplazo & \multirow{2}{*}{$\begin{array}{r}\text { Traducción español } \\
\text { Vellosidad coriónica primaria }\end{array}$} \\
\hline E6.0.2.5.0.1.7 & Gradus villosus in itialis & $\begin{array}{l}\text { Villitatis chorion prima, modificado de } \\
\text { Villus primarius (E6.0.1.2.0.0.15)* }\end{array}$ & \\
\hline E6.0.2.5.0.1.8 & Gradus labyrintheus & $\begin{array}{l}\text { Villitatis chorion secunda, modificado de } \\
\text { Villus secundarius (E6.0.1.2.0.0.17)* }\end{array}$ & Vellosidad coriónica secundaria \\
\hline E6.0.2.5.0.1.9 & Gradus villosus definitivus & $\begin{array}{l}\text { Villitat is chorion tertia, modificado de Villus } \\
\text { tertiarius (E6.0.1.2.0.0.20)* }\end{array}$ & Vellosidad coriónica terciaria \\
\hline E6.0.2.5.1.3.3 & Funiculus umbilicalis glomeratus & E6.0.2.2.0.0.1Funiculus umbilicalis & Cordón umbilical \\
\hline E6.0.2.5.1.3.8 & Vesicula Allantoica & E6.0.1.2.0.0.2 Allantois (griego) & Alantoides \\
\hline
\end{tabular}

Los términos incluidos en la Tabla V, corresponden a términos pertenecientes a otros capítulos de TE, y de acuerdo al contenido de los capítulos analizados en este trabajo, consideramos necesarios incluirlos, respetando su codificación original para no duplicar términos.

En la Tabla VI, se incluyen términos que deberían ser eliminados debido a que no son de uso habitual en la literatura de textos embriológicos y artículos científicos. Sin embargo, existen términos en otros capítulos de TE, que se correlacionan con los términos eliminados y que por lo tanto pueden reemplazarlos en los capítulos analizados en este trabajo.

Se destacan tres términos (con asterisco) que además deben ser modificados en el latín, ya que el término original no indicaba correctamente la estructura embriológica traducida al español. Estos nuevos términos mantendrán la misma codificación que el término en latín original.

\section{CONCLUSIÓN}

A partir del análisis de los capítulos indicados en este trabajo, se llevó a cabo la traducción del latín al español de los términos embriológicos relacionados con placenta humana. Hasta el momento, solo existe la traducción al inglés de TE, por lo tanto, a través de la traducción al español hemos querido aportar a unificar criterios en el uso de TE en nuestro idioma.

La traducción de los términos no fue idéntico, pues no se trató de realizar un calco lingüístico, sino una interpretación. Esto está en relación al sentido y la aplicación del término traducido.

A partir de nuestro análisis, identificamos lo siguiente:

- términos en latín, correctos gramatical y morfológicamente, y de los cuales hicimos la traducción al español

- términos en latín que requerían ser modificamos, y luego se tradujeron al español

- ausencia de ciertos términos en la TE, los cuales debimos crear en latín con su traducción al español

- términos no utilizados en la literatura consultada

- términos pertenecientes a otros capítulos que deberían ser incluídos en los capítulos analizados en este trabajo - términos del latín que deberían ser eliminados y reemplazados por términos ya existentes en otros capítulos de TE, y de los cuales realizamos su correspondiente traudcción al español.

Proponemos que los resultados alcanzados ern este trabajo sean dialogados con los miembros del grupo de trabajo de embriología del SILAT, para recibir las observaciones y comentarios de los expertos, contribuyendo a la validación de los resultados obtenidos y así realizar un aporte en español de TE que permita mejorar la comunicación entre los investigadores del área.

PRIETO, G. R. \& OTTONE, N. E. Terminologia embryologica and placenta: proposal of embryological terms in spanish. Int. J. Morphol., 36(1):63-68, 2018.

SUMMARY: In embryology, and in reference to the use of Terminologia Embryologica (TE), a number of terms used do not apply to TE., Since the origins of the Nomina Anatomica of Basel in 1895, the absence of embryological terms in TE is noted, even though these terms are commonly used in clinical practice. Furthermore, to date there is no official Spanish translation of TE. The aim of this work was to present a proposal of terms in Spanish, for the structures of the chapter Adnexa embryonica et fetalia; Membranae extraembryonicae et fetales corresponding to the terms included in Paraplacenta [E6.0.2.4.0.1.], Placenta [E5.11.3.1.1.0.5] and Anomaliae placentae [E6.0.2.5.1.0.1], from Terminologia Embryologica (TE) published by the Federal International Program on Anatomical Terminologies in 2013, wherein only the English translation can be found. The importance of all the articles related to the good use of terminologies and their correct translation into the vernacular language, is that the application of a unique and common language will allow a better and greater diffusion of the research in the area of the morphological sciences.

KEY WORDS: Terminologia Embryologica; Placenta. 


\section{REFERENCIAS BIBLIOGRÁFICAS}

Apaza Valencia, J. Desarrollo placentario temprano: aspectos fisiopatológicos. Rev. Peru. Ginecol. Obstet., 60(2):131-9, 2006.

Arteaga, M. \& García, M. I. Embriología Humana y Biología del Desarrollo. Ciudad de México, Editorial Panamericana, 2013.

Bernirschke, K. \& Kaufmann, P. Pathology of the human placenta. $4^{\mathrm{a}} \mathrm{ed}$. Nueva York, Springer-Verlag, 2000.

Bustamante-Zuluaga, C.; Parra-Anaya, G.; Díaz-Yunez, I.; Vergara-Quintero, F. \& De Nubbila-Lizcano, E. Pronóstico perinatal de los fetos con circular de cordón en relación con la vía del parto. Revisión de la literatura. Rev. Colomb. Obstet. Ginecol., 62(4) :315-20, 2011.

Carlson, B. M. Embriología Humana, y Biología del Desarrollo. $4^{\mathrm{a}}$ ed. Barcelona, Elsevier, 2009.

Cruz, G. R.; Rodríguez, T. A.; Prates, J. C.; Losardo, R. J. \& Prates, N. E. V. B. Ibero-Latin-American Symposium of Morphological Terminology. General Characteristics. Int. J. Morphol., 28(2):637-42, 2010.

Dudek, R. Embriología. $6^{a}$ ed. Barcelona, Lippincott Williams \& Wilkins Wolters Kluwer, 2014.

Federal International Programme on Anatomical Terminologies (FIPAT). Terminologia embryologica. International Embryological Terminology. Stuttgart, Thieme, 2013.

Flores, V. Embriología Humana. Bases moleculares y Celulares de la Histogénesis, la Morfogénesis y las Alteraciones del Desarrollo. Madrid, Editorial Médica Panamericana, 2015.

Huppertz, B. The anatomy of the normal placenta. J. Clin. Pathol., 61(12):1296-302, 2008

Losardo, R. J.; Cruz, G. R.; Rodríguez, T. A.; Prates, J. C. \& Prates, N. E. V. B. Iberia-Latin-American Symposia of Morphological Terminology (SILAT). First Two Years and Statute. Int. J. Morphol., 28(4):1323-6, 2010.

Losardo, R. J.; Prates, N. E. V. B.; Arteaga Martínez, M.; Cabral, R. H. \& García-Peláez, M. I. International Morphological Terminology: more than anatomy, histology and embryology. Int. J. Morphol., 33(1):4007, 2015.

Moore, K. L.; Perseaud, T. V. N. \& Torchia, M. G. Embriología Clínica. $11^{\mathrm{a}}$ ed. Barcelona, Elsevier, 2016.

Ottone, N. E.; Vargas, C. A. \& Del Sol, M. From omohyoid muscle to scapulohyoid muscle. Int. J. Morphol., 35(2):740-4, 2017.

Pérez Sánchez, A. \& Donoso, E. Obstetricia. $4^{a}$ ed. Santiago de Chile, Editorial Mediterráneo, 2011.

Prieto Gómez, R.; Smok, C. \& Rojas, M. Experiencias de blog: Placenta comparada. Int. J. Morphol., 29(2):432-5, 2011.

Roa, I.; Smok, C. \& Prieto Gómez, R. Placenta: Anatomía e histología comparada. Int. J. Morphol., 30(4):1490-6, 2012.

Sadler, T. W. Langman. Embriología Médica. 12ª ed. Barcelona, Lippincott
Williams \& Wilkins Wolters Kluwer, 2012.

Uzcátegui, M.; Rodríguez, A. M. \& Urdaneta, M. Circular de cordón umbilical irreductible en el feto y su relación con asfixia perinatal. Hosp. Niños J. M. de los Ríos, 41(1):27-32, 2005.

Vargas, C. A.; Ottone, N, E. Contreras, M. \& Del Sol, M. Facies or Impressio in the Spleen? Int. J. Morphol., 34(3):1002-8, 2016.

Vásquez, B. \& del Sol, M. Terminologia Anatomica y Terminologia Histologica: Un Lugar de Encuentro entre los Morfólogos. Int. J. Morphol., 33(4):1585-90, 2015.

\author{
Dirección para correspondencia \\ Ruth Prieto Gómez \\ Departamento de Pediatría y Cirugía Infantil \\ Facultad de Medicina \\ Universidad de La Frontera \\ Temuco \\ CHILE
}

E-mail: ruth.prieto@ufrontera.cl

Recibido : 20-07-2017

Aceptado: $13-10-2017$ 